\title{
Effects of Ashwagandha (Withania somnifera) Root Extract On Some Serum Liver Marker Enzymes (AST, ALT) In Gentamicin Intoxicated Rats
}

\author{
Nayma Sultana ${ }^{1}$, Sadia Choudhury Shimmi ${ }^{2}$, M.Tanveer Hossain Parash ${ }^{3}$, Jesmine Akhtar $^{4}$
}

\begin{abstract}
Background: Liver is an essential metabolic organ. It can be damaged due to prolonged use and higher doses of drugs, exposure to some chemicals, toxins, or infectious agents. Herbal plants as ashwagandha (Withania somnifera) may have free radical scavenging activity thereby can be used for the prevention and treatment of liver damage. Objective: To observe the effect of ashwagandha (Withania somnifera) root extract on gentamicin induced changes of some liver marker enzymes e,g serum aspartate amino transferase (AST) and alanine amino transferase (ALT) in Wistar albino rats. Methods: This experimental study was carried out in the Department of Physiology, Sir Salimullah Medical College (SSMC), Dhaka from $1^{\text {st }}$ July 2010 to $30^{\text {th }}$ June 2011. A total number of 35 Wistar albino rats, aged 90 to 120 days, weighing between 150 to 200 grams were selected for the study. After acclimatization for 14 days, they were divided into control group (Group A) and experimental group (Group B). Control group was again subdivided into group $\mathrm{A}_{1}$ (baseline control, consisted of 10 rats) and group $\mathrm{A}_{2}$ (gentamicin treated control group, consisted of 10 rats). Again, experimental group (Group B-ashwagandha pretreated and gentamicin treated group) consisted of 15 rats. All groups of animals received basal diet for 22 consecutive days. In addition to this, group $\mathrm{A}_{2}$ also received gentamicin subcutaneously ( $100 \mathrm{mg} / \mathrm{kg}$ body weight/day) for the last eight $\left(15^{\text {th }}\right.$ to $22^{\text {nd }}$ day) consecutive days. Again, group B received ashwagandha root extract $(500 \mathrm{mg} / \mathrm{kg}$ body weight/day, orally) for 22 consecutive days and gentamicin subcutaneously $\left(100 \mathrm{mg} / \mathrm{kg}\right.$ body weight $/$ day) for last eight ( $15^{\text {th }}$ to $22^{\text {nd }}$ day) days. All the animals were sacrificed on $23^{\text {rd }}$ day. Then blood and liver samples were collected. For assessment of liver function, serum AST, ALT and bilirubin levels were estimated. All these tests were done by standard Laboratory technique. The statistical analysis was done by one way ANOVA and Bonferroni test as applicable. Results: The mean serum levels of AST and ALT were significantly $(p<0.001)$ higher in gentamicin treated control group and in ashwagandha pretreated and gentamicin treated group in comparison to those of baseline control group. Again, these levels were significantly $(\mathrm{p}<0.001)$ lower in ashwagandha pretreated and gentamicin treated group than those of gentamicin treated control group. Conclusion: Ashwagandha (Withania somnifera) root extract restored serum AST, ALT towards normal levels in gentamicin intoxicated rats which may be due to its free radical scavenging activity. Therefore it may have hepatoprotective effect.
\end{abstract}

Key words: Heopatoprotective, Ashwagandha, Aspartate aminotransferase, Alanine aminotransferase

J Bangladesh Soc Physiol. 2012 June; 7(1): 1-7 For Authors Affiliation, see end of text.

http://www.banglajol.info/index.php/JBSP

$\overline{\text { Received December 2011; } \quad \text { Accepted March } 2012}$

J Bangladesh Soc Physiol. 2012, June; 7(1): 1-7 


\section{Introduction}

$\mathbf{T}$

he liver is the largest gland in the body.

It is essential for life because it conducts

vast array of biochemical and metabolic

functions, including ridding the body of substances that would otherwise be injurious if allowed to accumulate, and excreting drug metabolites ${ }^{1}$.

Liver is continuously exposed to a variety of chemicals, toxins, drugs etc ${ }^{2}$. Metabolism of these may cause metabolism-dependent, drug induced liver injury ${ }^{3}$. The drug metabolites can promote a variety of chemical reactions or inducing lipid peroxidation. All of these have consequent direct effects on cell organelles such as mitochondria, endoplasmic reticulum, the cytoskeleton microtubules, or the nucleus ${ }^{4}$. Drug-induced hepatotoxicity usually involves the participation of a toxic drug or metabolite that either elicits an immune response or directly affects functions of the cell ${ }^{5}$. Mitochondrial dysfunction is one of the major mechanism of drugs induce hepatotoxicity. By severely altering mitochondrial function in the liver, drugs can induce hepatic necrosis, causing cytolytic hepatitis, and can progress into liver failure ${ }^{7}$.

Gentamicin is a broad spectrum antibiotic belonging to the amino glycosides group which includes streptomycin, neomycin and kanamycin ${ }^{8}$. It is very effective in treating life threatening gram-negative bacterial and some gram positive bacterial infection ${ }^{9}$. The toxicity of gentamicin has been widely studied ${ }^{10}$ Gentamicin can cause nephrotoxicity, hepatotoxicity etc. ${ }^{11}$. This drug causes generation of reactive oxygen species (ROS), which induces cell injury and necrosis thus may produce liver toxicity ${ }^{12}$. Recently, different researchers ${ }^{11}$ from different countries observed the hepatotoxic effects of gentamicin. Again, some of the investigators found increased levels of some liver marker enzymes such as aspartate aminotransferase (AST), alanine aminotransferase (ALT), and billirubin after administration of toxic dose of gentamicin in rats ${ }^{11,13,14}$.
Withania somnifera, known as ashwagandha / Winter cherry/ Indian ginseng, an Ayurvedic medicine, belongs to the family of Solanaceae ${ }^{15}$. Different parts of this plant like Leaves, fruits, roots etc have therapeutic effects ${ }^{16}$. The roots of Withania somnifera contained 35 chemical constituents $^{17}$. Withaferin A and withanolides, the active ingredients contribute to the most of the biological actions of Withania ${ }^{18}$. Till today, no side effects have been found in ashwagandha $^{19}$.

Different researchers from different countries have been studied the hepatoprotective effects of ashwagandha (Withania somnifera) ${ }^{14}$. Recently, some investigators observed that ashwagandha significantly decreased the toxic induced elevation of serum AST, ALT and bilirubin levels in rats $16,20,21$.

Liver failure is one of the important issue in our country. In absence of reliable hepatoprotective drug in modern medicine, Auervedic medicinal preperation can be used as hepatoprotective agents. But in Bangladesh, little is known about the effects of ashwagandha (Withania somnifera). Ashwagandha is reasonably cheap, available and relatively safe which is cultivated and harvested in some Ayurvedic nursery of Bangladesh. Therefore, the present study was carried out to observe the effects of ashwagandha (Withania somnifera) root extract on some serum liver marker enzymes (AST, ALT) in gentamicin intoxicated rats. It is also expected that the result of this study would make the ashwagandha acceptable among the people as a rich source with medicinal value for the prevention of liver damage.

\section{Methods}

This experimental study was conducted between July 2010 to June 2011 in the Department of Physiology, SSMC, Mitford, Dhaka. A total number of 35 apparently healthy Wistar albino male rats, weighing between 150 to 200 grams, age range 90 to 120 days were used. The animals

J Bangladesh Soc Physiol. 2012, June; 7(1): 1-7 
were purchased from animal house of Bangladesh Council of Scientific and Industrial Research (BCSIR), Dhaka.. Ethical permission was taken from the Institutional Ethics Committee (IEC) of SSMC, Dhaka. Prior conducting the study, the animals were acclimatized for 14 days at $23 \pm 2^{0} \mathrm{C}$ room temperature under 12 hour dark- light cycle. During this period, they had free access to food and water ad libitum. Then they were divided into control group (Group A) and experimental group (Group B). Control group was again subdivided into baseline control, consisted of 10 rats (group $\mathrm{A}_{1}$ ) and gentamicin treated control group, consisted of $10 \operatorname{rats}\left(\right.$ group $\left.A_{2}\right)$. The experimental group consisted of 15 rats (Group B).They were Ashwagandha pretreated and gentamicin treated.. All groups of animals received basal diet for 22 consecutive days. In addition to this, group $\mathrm{A}_{2}$ also received gentamicin subcutaneously (100mg $/ \mathrm{kg}$ body weight/day) for the last eight (15 th $22^{\text {nd }}$ day) consecutive days. Again, group B received ashwagandha root extract $(500 \mathrm{mg} / \mathrm{kg}$ body weight/day, orally) for 22 consecutive days and gentamicin subcutaneously $(100 \mathrm{mg} / \mathrm{kg}$ body weight /day) for last eight ( $15^{\text {th }}$ to $22^{\text {nd }}$ day) days. After giving gentamicin and ashwagandha all the animals including baseline control rats, were anaesthetized with the help of chloroform and sacrificed on $23^{\text {rd }}$ day. After 14 days of acclimatization, body weights were measured (initial bw), Again, final body weights (final bw) of rats before anaesthetized on $23^{\text {rd }}$ day were taken. The blood and liver samples were collected. Liver was washed in ice cold saline. Then, it was wiped in tissue paper and weighed by electric balance analyzer. Blood was centrifuged at the rate of $4000 \mathrm{rpm}$ for 5 minutes and supernatant serum was collected. Out of 35 serum samples, four (4) were hemolyzed. So, thirty one (31) samples were estimated. Then, for assessment of liver function some liver marker enzymes e,g serum AST and ALT and serum bilirubin levels were estimated. All these tests were done by standard laboratory technique 22,23 in the laboratory of Department of Physiology and Biochemistry, SSMC.

J Bangladesh Soc Physiol. 2012, June; 7(1): 1-7
Statistical analysis were done by one way ANOVA and Bonferroni test by using SPSS windows, version 16.

Preparation of root extract of ashwagandha (Withania somnifera):

Ashwagandha is cultivated and harvested in the Ayurvedic nursery of Hamdard Laboratories, Meghna. After collecting the roots of this plant, it was dried in sunlight for 2 days, crushed in an electrical grinder into powder. Then the powder was extracted in methanol, filtered, evaporated by rotatory evaporator and dried. After that, the dried root extract of ashwagandha was dissolved by propylene glycol $(2 \mathrm{ml} / \mathrm{kg}$ body weight $)$ and finally mixed with distilled water for feeding.

\section{Results}

Body weights of the rats are presented in Table I.

The liver weight was significantly $(\mathrm{p}<0.01)$ higher in gentamicin treated control group (group $\mathrm{A}_{2}$ ) when compared to that of baseline control (group $A_{1}$ ) and ashwagandha pretreated and gentamicin treated group ( group B). Again, liver weight of ashwagandha pretreated and gentamicin treated group (group B) and baseline control group (group $\mathrm{A}_{1}$ ) was almost similar and no statistically significant difference of liver weight was observed between the groups (Table I).

The mean serum levels of AST and ALT were significantly higher in gentamicin treated control (group $\mathrm{A}_{2} \mathrm{p}<0.001$ ) and ashwagandha pretreated and gentamicin treated group (group $\mathrm{B} p<0.05$ ) in comparison to those of baseline control group (group $\mathrm{A}_{1}$ ) Again, these levels of ashwagandha pretreated and gentamicin treated group (group B) were significantly $(\mathrm{p}<0.001)$ lower than those of gentamicin treated control group (group $\mathrm{A}_{2}$ ) (Table II).

Again, the serum level of bilirubin was significantly higher in gentamicin treated control group (group $\mathrm{A}_{2}, \mathrm{p}<0.001$ ) and ashwagandha pretreated and gentamicin treated group (group $\mathrm{B}(\mathrm{p}<0.01)$ in comparison to those of baseline control (group $A_{1}$ ) Again, this level of ashwagandha pretreated and gentamicin treated group (group B) was significantly $(\mathrm{p}<0.01)$ lower than that of gentamicin treated control group (group $\mathrm{A}_{2}$ ). (Figure 1). 
Table I: Body weight and liver weight in different groups $(\mathrm{n}=31)$

\begin{tabular}{|c|c|c|c|c|}
\hline \multirow[t]{2}{*}{ Groups } & \multicolumn{2}{|c|}{ Body weight (g) } & \multirow{2}{*}{$\begin{array}{c}\% \text { of change from final }(\mathrm{F}) \\
\text { weight to initial }(\mathrm{I}) \\
\text { weight }[(\mathrm{F}-\mathrm{I}) / \mathrm{I} \times 100]\end{array}$} & \multirow{2}{*}{$\begin{array}{l}\text { Liver weigh } \\
\text { (g) }\end{array}$} \\
\hline & Initial (Day 1) & Final (Day 23) & & \\
\hline $\mathrm{A}_{1}$ & $181.13 \pm 2.64$ & $187.18 \pm 3.66$ & $3.34 \pm 0.92$ & $6.93 \pm 0.07$ \\
\hline$(\mathrm{n}=9)$ & $(177.12-185.24)$ & (183.10-194.09) & $(1.92-4.38)$ & $(6.82-7.01)$ \\
\hline $\mathrm{A}_{2}(\mathrm{n}=9)$ & $\begin{array}{c}182.30 \pm 2.80 \\
(178.04-185.92)\end{array}$ & $\begin{array}{c}176.83 \pm 3.69 \\
(169.67-180.28)\end{array}$ & $\begin{array}{c}-2.99 \pm 1.47 \\
-0.64 \text { to }-4.92\end{array}$ & $\begin{array}{l}7.33 \pm 0.47 \\
(6.89-7.99)\end{array}$ \\
\hline $\mathrm{B}(\mathrm{n}=13)$ & $181.85 \pm 3.13$ & $180.46 \pm 2.59$ & $-0.75 \pm 0.79$ & $6.79 \pm 0.29$ \\
\hline & $(177.10-186.92)$ & $(175.92-184.26)$ & -0.06 to -2.02 & $(5.92-7.1)$ \\
\hline \multicolumn{5}{|c|}{ Statistical analysis } \\
\hline & \multicolumn{4}{|c|}{$\mathrm{P}$ value } \\
\hline$A_{1}$ vs $A_{2}$ & $0.189^{\mathrm{ns}}$ & $0.000^{* * *}$ & $0.001^{* *}$ & $0.001^{* *}$ \\
\hline$A_{1}$ vs $B$ & $0.285^{\mathrm{ns}}$ & $0.000^{* * *}$ & $0.004^{* *}$ & $0.056^{\mathrm{ns}}$ \\
\hline $\mathrm{A}_{2}$ vs $\mathrm{B}$ & $0.363^{\text {ns }}$ & $0.012^{*}$ & $0.003^{\text {** }}$ & $0.005^{* *}$ \\
\hline
\end{tabular}

Results are expressed as Mean $\pm \mathrm{SD}$. Statistical analysis was done by ANOVA test and then Bonferroni test was performed to compare between groups. Figures in parentheses indicate ranges.Group $\mathrm{A}_{1}$ (Baseline control group), Group $\mathrm{A}_{2}$ (Gentamicin treated control group), Group B (Ashwagandha pretreated and gentamicin treated group) $\mathrm{n}$ : Number of subjects ns: Non-significant *: Significant at $\mathrm{p}<0.05 * *$ : Significant at $\mathrm{p}<0.01 * * *$ : Significant at $\mathrm{p}<$ 0.001

Table II: Serum AST and ALT levels in different groups $(\mathrm{n}=31)$

\begin{tabular}{lccc}
\hline Group & $\mathrm{n}$ & AST (IU/L) & ALT(IU/L) \\
\hline $\mathrm{A}_{1}$ & 9 & $47.33 \pm 3.74$ & $50.11 \pm 2.47$ \\
& & $(43.59-51.07)$ & $(47.64-52.58)$ \\
$\mathrm{A}_{2}$ & 9 & $90.89 \pm 4.53$ & $99.11 \pm 5.26$ \\
& & $(86.36-95.42)$ & $(93.85-104.37)$ \\
$\mathrm{B}$ & 13 & $50.62 \pm 2.14$ & $54.69 \pm 2.93$ \\
& & $(52.76-48.48)$ & $(51.76-57.62)$ \\
\hline
\end{tabular}

Statistical analysis

\begin{tabular}{lcc}
\hline \multicolumn{3}{c}{ P value } \\
\hline $\mathrm{A}_{1}$ vs $\mathrm{A}_{2}$ & $0.000^{* * *}$ & $0.000^{* * * *}$ \\
$\mathrm{~A}_{1}$ vs B & $0.035^{*}$ & $0.021^{*}$ \\
$\mathrm{~A}_{2}$ vs B & $0.000^{* * *}$ & $0.000^{* * *}$ \\
\hline
\end{tabular}

Results are expressed as Mean $\pm \mathrm{SD}$. Statistical analysis was done by ANOVA test and then Bonferroni test was performed to compare between groups. Figures in parentheses indicate ranges. Group $\mathrm{A}_{1}$ (Baseline control group). Group $\mathrm{A}_{2}$ (Gentamicin treated control group) Group B (Ashwagandha pretreated and gentamicin treated group)

$\mathrm{n}$ : Number of subjects $*$ : $<<0.05 * * *: \mathrm{p}<0.001$

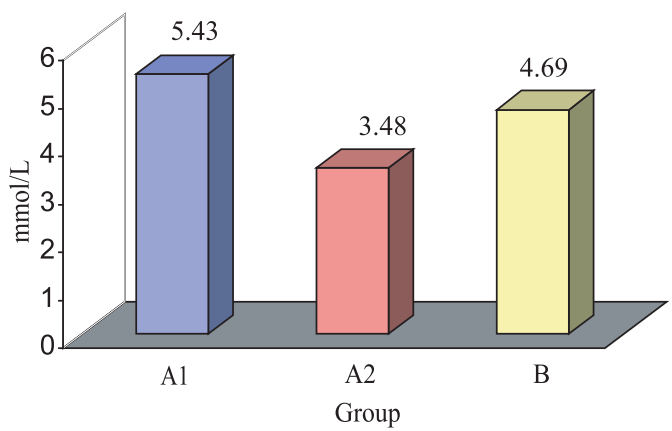

Figure 1: Serum bilirubin levels in different groups of rats $(n=31)$

\section{Discussion}

In this study, the liver weight was significantly higher in gentamicin treated control rats when compared to that of baseline control and ashwagandha pretreated and gentamicin treated group. But, no significant difference of this value was observed between ashwagandha pretreated and gentamicin treated group and baseline control group in this study. This finding is in consistent with that of some other investigators ${ }^{11}$.

J Bangladesh Soc Physiol. 2012, June; 7(1): 1-7 
In the present study, serum levels of AST and ALT were significantly higher in gentamicin treated control and in ashwagandha pretreated and gentamicin treated group in comparison to those of baseline control group. Again, significantly lower levels of

these parameters were observed in ashwagandha pretreated and gentamicin treated group when compared to those of gentamicin treated control group. Similar findings were also reported by different researchers ${ }^{11,14}$.

The serum level of bilirubin was significantly higher in gentamicin treated control group and in ashwagandha pretreated and gentamicin treated group in comparison to those of baseline control group. Whereas, significantly lower level of this parameter was observed in ashwagandha pretreated and gentamicin treated group when compared to that of gentamicin treated control group. Similar findings were also reported by different researchers ${ }^{11,13}$.

It has been suggested that, toxic dose of gentamicin may decrease the plasma magnesium concentration. This hypomagnesaemia may reduce the body weight, as magnesium is an essential mineral required for regulation of carbohydrate, protein and lipid metabolism and for maintenance of normal health ${ }^{25}$.

However, gentamicin induced toxicity causes oedema and inflammatory changes in the hepatic cells and thereby increases the liver weight ${ }^{11}$. Again, Gentamicin in toxic doses causes free radical generation and alteration in antioxidant enzyme activities which may be responsible for liver tissue injury and ultimately may influence verious metabolic pathway of liver ${ }^{11} 13$. Moreover, gentamicin toxicity alters cell membrane permeability and causes release of AST and ALT from the cell thus leads to increased serum concentration of these enzymes which are generally considered as sensitive markers of liver function ${ }^{13}$.

J Bangladesh Soc Physiol. 2012, June; 7(1): 1-7
However, some reseaschers suggested that, ashwagandha root contains sitoindosides VII$\mathrm{X}$ and withaferin $\mathrm{A}$, have antioxidant activity by enhancing the free radical scavenging enzymes such as, superoxide dismutase (SOD), catalase (CAT), glutathione peroxidase (GPx $)^{15,18}$. So, it may suppress toxin mediated enhancement of serum AST, ALT and bilirubin levels ${ }^{14,20,21}$. Again, ashwagandha root extract contains withanolides, which have anti-inflammatory property, thus may be helpful in protecting the liver damage and can decrease its weight ${ }^{26}$.

In the present study, hepatic damage was observed in rats treated with gentamicin as evidenced by their elevated levels of serum AST, ALT and bilirubin. This is further supported by an increase in liver weight in the same group of animal of this study.

Again, significantly lower levels of serum ASTand ALT which come almost normal level in the ashwagandha pretreated and gentamicin treated rats provides an evidence that, this root extract may have hepatoprotective effects against gentamicin toxicity. This is further supported by significantly lower levels of serum bilirubin levels and liver weights in this group of animals. All these effects are most likely due to presence of some active ingredients in ashwagandha root which have antioxidant property. However, the exact mechanism involved cannot be elucidated from this type of study due to time and financial constrains.

\section{Conclusion}

From this study, it can be concluded that ashwagandha (Withania somnifera) root extract may have some role in decreasing serum AST, ALT towards normal levels in gentamicin intoxicated rats may be due to its free radical scavenging activity which reflecting its hepatoprotective effect.

\section{Acknowledgement}

Authors of this study are thankful to the authority of Pharmacy Department of Dhaka University, 
Hamdard Laboratories and BCSIR, Dhaka for the cooperation they provided. I would also express my sincere thanks to the DGHS for providing the partial financial support for completing the research work.

\section{Author Affiliations}

1. * Nayma Sultana, Associate Professor, Department of Physiology, Sir Salimullah Medical College, Dhaka. Email : nayma_sultana@yahoo.com

2. Sadia Choudhury Shimmi, Assistant Professor, Department of Physiology, Delta Medical College and Hospital, Dhaka. E-mail: shimmi_cmc 40@yahoo.com

3. M.Tanveer Hossain Parash. Assistant Professor, Department of Anatomy, Satkhira Medical College. E-mail: parash_cmc@yahoo.com

4. Jesmine Akhtar. Associate Professor, Department of Physiology, East West Medical College, Dhaka.

*for correspondence

\section{References}

1. Pundir R, Singh G, Pandey AA, Saraf SA. Demand of herbal hepatoprotective formulations in Lucknow - a survey. The Pharma Research 2009; 01:23-33.

2. Sakr SA, Samai HAA, Soliman ME. Exploring hepatotoxicity of benomyl:histological and histochemical study on albino rats.J Med Sci 2004; 4(1):77-83.

3. Kaplowitz N. Mechanisms of liver cell injury. J Hepatol 2000; 32:39-47.

4. Lee WM. Drug induced Hepatotoxicity.N Engl J Med 2003; 349:474-85.

5. Kaplowitz N. Drug induced liver injury. CID 2004;38 (2):44-48.

6. Halt MP, Ju C. Mechanisms of drug induced liver injury. AAPS J 2006; 8 (1):48-54

7. Labbea G, Pessayreb D, Fromentyb B. Drug-induced liver injury through mitochondrial dysfunction: mechanisms and detection during preclinical safety studies. Fundamental \& Clinical Pharmacology 2008; 22: 335-353.

8. Gingell JC, Water worth PM. Dose of gentamicin in patients with normal renal function and renal impairment. Brit Med J 1968; 2: 19-22.
9. Yasin KF, Sabir M, Sherwani MIK, Zafar M, Yasmin $\mathrm{S}$, Alam MI. Amelioration of gentamicin nephrotoxicity by vitamin $\mathrm{B}_{6}$ (a general and biochemical profile). Pakistan J Med Res 2003; 42(2): 69-73

10. Klemens JJ, Meech RP and Hughes LF. Antioxidant enzyme levels inversely co vary with hearing loss after amikacin treatment.J Am Acad Audiol 2003; 14:134-42.

11. Noorani AA, Gupta KA, Bhadada K, Kale MK. Protective Effect of Methanolic Leaf Extract of Caesalpinia Bonduc (L.) On Gentamicin-Induced Hepatotoxicity and Nephrotoxicity in Rats. IJPT 2011; 10: 21-25

12. Aubrecht J, Goad MEP, Simpson EM. Expression of hygr in Transgenic Mice Causes Resistance to Toxic Effects of Hygromycin B In Vivo. J Pharmacol Exp Ther 1997; 281:992-7.

13. Khan MR, Badar I, Siddiquah A. Prevention of hepatorenal toxicity with Sonchus asper in gentamicin treated rats. BMC Complementary and Alternative Medicine 2011; 11:113-121.

14. Elberry AA, Harraz FM, Ghareib SA, Nagy AA, Gabr SA, Suliaman MI, and Abdel-Sattar E. Antihepatotoxic effect of Marrubium vulgare and Withania somnifera extracts on carbon Tetrachloride-induced hepatotoxicity in rats. JBCP 2010; 1(4): 247-254.

15. Mishra LC. Scientific Basis for the therapeutic use of Withania somnifera (Ashwagandha): a review. Altern Med Rev 2000; 5(4): 334-346.

16. Widodo N, Kaur K, Shrestha BG, Takagi Y, Ishii T, Renu W, Kaul SC. Selective killing of cancer cells by leaf extract of Ashwagandha: identification of a tumor inhibitory factor and the first molecular insights to its effect. Clin Cancer Res 2007; 13(7): 2298-2306.

17. Rastogi RP, Mehrotra BN. Compendium of Indian Medicinal plants. Central drug Research Institute 1998; 6:16.

18. Singh G, Sharma PK, Singh S. Biological activities of Withania somnifera. Annals of Biological research 2010;1(3): 56-63.

19. Das K, Samanta TT, Samanta P, Nandi DK. Effect of extract of Withania somnifera on dehydration -induced oxidative stress- related uremia in male rats. Saudi J of Kidney dis Transpl 2010; 21(1): 7580 .

J Bangladesh Soc Physiol. 2012, June; 7(1): 1-7 
20. Harikrishnan B, Subramanian P, Subash S. Effect of Withania somnifera root powder on the levels of circulatory lipid peroxidation and liver marker enzymes in chronic Hyperammonemia. E- Journal of Chemistry [Internet]. 2008 Oct [cited 2011 Jun 9]; 5(4): 872-877.Available from: http://www.ejournal.net

21. Borde AU, Qureshi MI, Patil MK, Mendhe MS, Athawale AM. Protective effect of Withania Somnifera on cadmium chloride induced hematological and biochemical changes in male rats. J Res Educ Indian Med 2008; 15(2): 15-20.

22. Bergmeyer HU, Horder M, Rej R. J. Clin Chem Clin Biochem 1986; 24: 481-495.
23. Rand RN, Di pasqua A. Clin. Chem. 1962; 8: 570.

24. Kays SE, Crowell WA, Johnson MA. Iron supplementation increases gentamicin nephrotoxicity in rats. J. Nutr 1991; 121: 18691875 .

25. Whang R. Magnesium deficiency, causes and clinical implications. Drugs 1984; 1: 143-150.

26. Ichikawa H, Takada Y, Shishodia S, Jayaprakasham B, Nair MG, Aggarwal BB. Withanolides potentiate apoptosis, inhibits invasion and abolish osteoclastogenesis through suppression of nuclear factor-kappa B (NF- $\sim$ B) activation and NF-kappa B- regulated gene expression. Mol Cancer Ther 2006; 5(6): 1434-1445. 